\title{
Berufliche Rehabilitation: First Place, then Train - Pro
}

\author{
Vocational Rehabilitation: First Place, then Train - Pro
}

\section{Pro}

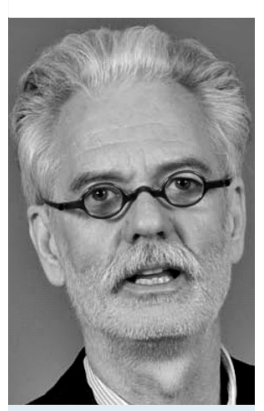

Holger Hoffmann
Teilhabe am Arbeitsleben und soziale Inklusion sind seit jeher wichtige Rehabilitationsziele für Menschen mit psychischen Störungen. Herkömmliche berufliche Wiedereingliederungsmaßnahmen funktionieren nach dem Prinzip „erst trainieren - dann platzieren“, d.h. es findet zunächst ein vorbereitendes Arbeitstraining im geschützten Rahmen statt, um dann bei ausreichenden Trainingseffekten eine Arbeitsstelle zu suchen. Diese Maßnahmen haben erwiesenermaßen geringe Erfolgsquoten bei der Wiedereingliederung schwer und anhaltend psychisch Kranker. Der größte Teil der Wiedereinzugliedernden ist nach $\mathrm{Ab}$ schluss der Maßnahme arbeitslos, in einer geschützten Werkstatt beschäftigt oder befindet sich in einer weiteren Schleife der Rehabilitation. Lediglich 5-30\% erreichen das Ziel einer Anstellung auf dem allgemeinen Arbeitsmarkt, und von diesen verliert wiederum ein beträchtlicher Teil ihre Stelle innerhalb Jahresfrist. Wir dürfen heute davon ausgehen, dass lediglich rund $15 \%$ nachhaltig über diesen Weg wiedereingegliedert werden können [1]. Es stellt sich somit immer dringender die Frage, ob die bisherigen Rehabilitationskonzepte noch zielführend sind.

Mit der Einführung des Supported Employment Anfang der 90er-Jahre in den USA, das nach dem Prinzip „erst platzieren - dann trainieren“ konzipiert ist, wurde in der beruflichen Wiedereingliederung psychisch beeinträchtigter Menschen ein Paradigmawechsel vollzogen, der sich nicht nur in den USA, sondern mittlerweile auch in Europa bewährt hat. Die wissenschaftliche Evidenz für die Überlegenheit von Supported Employment konnte mittels mittlerweile über 15 weltweit durchgeführter randomisiert kontrollierter Studien erhärtet werden [2].

Eine wichtige Studie in diesen Zusammenhang ist die Multicenterstudie EQOLISE [3], die sich zum Ziel gesetzt hatte, in 6 europäischen Ländern mit unterschiedlichen Arbeitsmarktbedingungen, Arbeitslosenraten und Sozialversicherungssystemen die amerikanischen Studien zu replizieren. Es gab jedoch unter den teilnehmenden Ländern Unterschiede, so schnitten die Zentren in Holland, Deutschland und die Schweiz tendenziell schlechter ab als die in England, Italien und Bulgarien. Die Tatsache, dass das Supported Employment im deutschen Zentrum keine signifikant höheren Eingliederungsquoten erzielte als die als Kontrollmaßnahme dienende Einrichtung, gab sowohl bei der Erstellung der „Praxisleitlinien Rehabilitation für Menschen mit psychischen Störungen“ [4], als auch bei der „S3-Leitlinie Psychosoziale Therapien bei Menschen mit schweren psychischen Erkrankungen“ [5] Anlass zu kontroversen Diskussionen bezüglich der Wirksamkeit von Supported Employment im deutschsprachigen Raum. In der S3-Leitlinie wurde folgende Empfehlung (No.12) gegeben: „Zur beruflichen Rehabilitation von Menschen mit schweren psychischen Erkrankungen, die eine Tätigkeit auf dem ersten Arbeitsmarkt anstreben, sollen Programme mit einer raschen Platzierung direkt auf einen Arbeitsplatz des ersten Arbeitsmarktes und unterstützendem Training (Supported Employment) genutzt und ausgebaut werden." Nachdem man sich zunächst mehrheitlich für Empfehlungsgrad A mit Evidenzebene la geeinigt hatte, wurde im Rahmen des Verabschiedungsprozesses von den Vorständen der Fachgesellschaften kritisch hinterfragt, ob sich Supported Employment tatsächlich auf das deutsche Versorgungssystem übertragen lasse. Eine entsprechende randomisierte kontrollierte Studie stehe noch aus, weshalb der Empfehlungsgrad auf B herabgestuft wurde [5].
Ebenfalls mit Empfehlungsgrad B, jedoch auf Evidenzebene Ib wurde eine zweite Empfehlung (No.13) abgegeben: „Zur Förderung der Teilhabe schwer psychisch kranker Menschen am Arbeitsleben sollten auch Angebote vorgehalten werden, die nach dem Prinzip „erst trainieren, dann platzieren" vorgehen. Diese sind insbesondere für die Teilgruppe schwer psychisch Kranker unverzichtbar, für die eine Platzierung auf dem ersten Arbeitsmarkt (noch) kein realistisches Ziel darstellt. Finanzielle Anreize erhöhen die Wirksamkeit entsprechender Angebote. Die Kombination der Angebote mit Interventionen, die auf Motivationssteigerung abzielen, oder ein rasches Überleiten der Programmteilnehmer in bezahlte übergangsweise Beschäftigung, erhöht ebenfalls die Wirksamkeit“ [5].

Diese Empfehlung irritiert. Einerseits erhalten die traditionellen Programme nach dem Prinzip „erst trainieren, dann platzieren“ den gleichen Empfehlungsgrad wie das Supported Employment, obwohl sie sich dem Supported Employment als unterlegen erwiesen haben. Andererseits ist Supported Employment nicht in erster Linie für die leistungsfähigeren und sozial kompetenteren Rehabilitanden konzipiert, sondern bringt gerade diejenigen zurück auf den ersten Arbeitsmarkt, die dieses Ziel in den traditionellen Wiedereingliederungsmaßnahmen trotz längeren Trainings nicht erreichen [6, 7]. Die Kontroverse um den Empfehlungsgrad fand eine erste Beruhigung mit der Publikation der 2-Jahres-Ergebnisse unserer randomisiert kontrollierten Studie zum Berner Job Coach Projekt [8]. Nach 2 Jahren arbeiten $45 \%$ der Teilnehmer in der freien Wirtschaft gegenüber $17 \%$, die ein herkömmliches Trainingsprogramm durchlaufen haben. Mit der weltweit erstmals durchgeführten kontrolliert-randomisierten 5-Jahres-Verlaufsstudie konnten wir zeigen, dass die Unterschiede gegenüber den herkömmlichen beruflichen Wiedereingliederungsmaßnahmen noch deutlicher werden als nach 2 Jahren [1]. So fanden in diesem Zeitraum 65\% der 
von einem Job Coach begleiteten TeilnehmerInnen in diesem Zeitraum eine Anstellung auf dem allgemeinen Arbeitsmarkt gegenüber 33\% in den als Kontrollgruppe dienenden traditionellen Programmen. Auch waren der Verdienst besser, die Anstellungsverhältnisse länger und nachhaltiger und die Inanspruchnahme (teil-)stationärer psychiatrischer Dienste geringer. Auf der finanziellen Ebene erwies sich der Social Return on Investment beim Supported Employment als deutlich höher d.h. es ist auch ökonomisch überlegen.

Diese eindrücklichen Ergebnisse - die sich problemlos von der Schweiz auf Deutschland übertragen lassen - unterstreichen, dass Supported Employment im deutschsprachigen Raum als Methode der Wahl bei der beruflichen Wiedereingliederung psychisch kranker Menschen vorbehaltlos empfohlen werden und gegenüber den weniger effektiven traditionellen Wiedereingliederungsprogrammen der Vorzug gegeben werden sollte.

Viele in der beruflichen Rehabilitation Verantwortlichen sind noch dem Stufenleitermodell traditioneller Rehabilitationskonzepte verhaftet und lassen sich von der Annahme leiten, dass ein schrittweise aufbauendes Arbeitstraining im geschützten Rahmen die RehabilitandInnen auf die Arbeit in der freien Wirtschaft vorbereite und damit die Wiedereingliederungschancen erhöhe. Entsprechend schwer vorstellbar ist, dass es gerade bei vermeintlich schwierigen RehabilitandInnen auch ohne ein vorangehendes Train- ing im geschützten Rahmen die Wiedereingliederung sogar besser gelingen könnte. Zudem wird das Supported Employment als eine Bedrohung und Entwertung der meist seit Jahrzehnten bestehenden Einrichtungen und der dort geleisteten Arbeit erlebt. Dies löst Widerstände und ein Lobbying für den Erhalt des Bewährten aus, verhindert erfreulicherweise jedoch nicht, dass die Zahl der Einrichtungen, die Supported Employment anbieten, in Deutschland, Österreich und der Schweiz ständig zunimmt. Wenn es gelingt, in nützlicher Frist, einheitliche Qualitätsstandards zu erarbeiten, attraktive Anreize für die Arbeitgeber zu schaffen und Finanzierungsmodelle, die eine zeitlich unbefristete Begleitung durch einen Job Coach ermöglichen, dann ist der Siegeszug des Supported Employment auch im deutschsprachigen Raum kaum noch zu bremsen.

\section{Interessenkonflikt \\ $\nabla$}

Der Autor ist Wissenschaftlicher Beirat von Supported Employment Schweiz.

\section{Literatur}

1 Hoffmann H, Jäckel D, Glauser S et al. Longterm effectiveness of supported employment: Five-year follow-up of a randomized controlled trial. Am J Psychiatry 2014 (in Druck)

2 Bond GR, Drake RE, Becker DR. Generalizability of the Individual Placement and Support (IPS) model of supported employment outside the US. World Psychiatry 2012; 11 : $32-39$
3 Burns T, Catty J, Becker T et al. The effectiveness of supported employment for people with severe mental illness: a randomised controlled trial. Lancet 2007; 370: $1146-$ 1152

4 Jäckel D, Hoffmann H, Weig W. Praxisleitlinien Rehabilitation für Menschen mit psychischen Störungen. Bonn: Psychiatrie Verlag; 2010

5 DGPPN, Hrsg. S3-Leitlinie Psychosoziale Therapien bei schweren psychischen Erkrankungen. Heidelberg: Springer; 2013

6 Brieger P, Hoffmann $H$. Was bringt psychisch Kranke besser und nachhaltiger in Arbeit? Nervenarzt 2012; 83: 840-846

7 Hoffmann H. Was macht Supported Employment so überlegen? Die Psychiatrie 2013; 10: $95-101$

8 Hoffmann H, Jäckel D, Glauser S et al. A randomised controlled trial of the efficacy of supported employment. Acta Psychiatr Scand 2012; 125: 157-167

Sie haben eine eigene Meinung zu diesem Thema? Dann schreiben Sie uns an: psychiat-praxis@thieme.de!

Korrespondenzadresse PD Dr. med. Holger Hoffmann Universitäre Psychiatrische Dienste Bern, Direktion Psychiatrische Rehabilitation Murtenstraße 46

3000 Bern 10, Schweiz

hoffmann@spk.unibe.ch

Bibliografie

DOI http://dx.doi.org/

10.1055/s-0034-1370232

Psychiat Prax 2014; 41: 293-294

(c) Georg Thieme Verlag KG

Stuttgart · New York

ISSN 0303-4259 